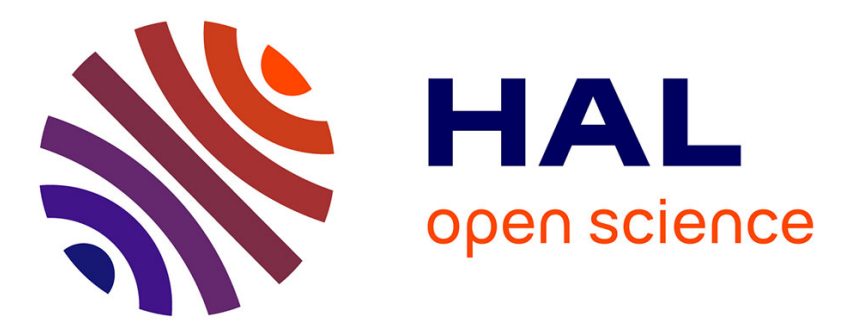

\title{
3-D Imaging of a Microwave Absorber Sample From Microwave Scattered Field Measurements
}

Jean-Michel Geffrin, Christelle Eyraud, Amelie Litman

\section{To cite this version:}

Jean-Michel Geffrin, Christelle Eyraud, Amelie Litman. 3-D Imaging of a Microwave Absorber Sample From Microwave Scattered Field Measurements. IEEE Microwave and Wireless Components Letters, 2015, 25 (7), pp.472 - 474. 10.1109/LMWC.2015.2427577 . hal-01279547

\section{HAL Id: hal-01279547 \\ https://hal-amu.archives-ouvertes.fr/hal-01279547}

Submitted on 31 Oct 2018

HAL is a multi-disciplinary open access archive for the deposit and dissemination of scientific research documents, whether they are published or not. The documents may come from teaching and research institutions in France or abroad, or from public or private research centers.
L'archive ouverte pluridisciplinaire HAL, est destinée au dépôt et à la diffusion de documents scientifiques de niveau recherche, publiés ou non, émanant des établissements d'enseignement et de recherche français ou étrangers, des laboratoires publics ou privés.

\section{(c)(1)}

Distributed under a Creative Commons Attribution| 4.0 International License 


\title{
3D Imaging Of A Microwave Absorber Sample From Microwave Scattered Field Measurements.
}

\author{
Jean-Michel Geffrin, Christelle Eyraud, Amélie Litman
}

\begin{abstract}
The internal structure of a sample of an absorber is retrieved from its measured scattered field. By its very nature, this target is a very weak-scatterer as it is used to cover the walls in anechoic chambers. The real part of its permittivity is rather small but its dielectric losses are non negligible. Quantitative maps of the complex permittivity of the sample were obtained from monochromatic scattered fields measured inside an anechoic chamber in a multistatic configuration. An imaging procedure taking into account the noise characteristics disturbing the measurements was used for this purpose.
\end{abstract}

Index Terms-Inverse problems, Microwave imaging, Anechoic chamber, Calibration, Vector Network Analyzer (VNA), Multistatic radar, Dielectric losses

\section{INTRODUCTION}

For several years, microwave imaging has attracted great interest in the research community as it intervenes in several practical applications, for example, biomedical imaging, subsurface imaging and non-destructive testing. Nevertheless, there is still a significant step to make in order to achieve quantitative three-dimensional microwave imaging of natural structures. With this in mind, recent developments concern the extension and acceleration of inverse scattering algorithms to tackle 3D targets [1]-[4]. At the same time, progresses on the measurements of scattered fields allow now to accurately measure 3D structures in free space [5]-[7] or in confined microwave systems. There is still few contributions concerning 3D quantitative imaging from experimental data due to the sensitivity of such algorithms [6], [9]-[12]. In addition, until now, laboratory conditions are needed to acquire such measurements due to the requested accuracy.

To test the efficiency of our experimental [5] [7] and numerical developments [8] in a borderline case, we have selected a component that every microwave experimenter has been working with: the extremity of a microwave absorbing pyramid. With this target, we face major difficulties: it is a low scattering target, large with respect to the wavelength, eventually heterogeneous and with a finite loss tangent worth to be accurately determined. At least to our knowledge, it is the first time that a microwave absorber is quantitatively and volumetrically imaged. Such imaging is of great interest to perform a non-destructive evaluation of the inner composition of the absorber samples or of any lossy sample.

Achieving such three-dimensional quantitative reconstructions requires a certain level of maturity of the entire instrumental chain. Indeed, particular attention must be paid

All the authors are with Aix Marseille Université, CNRS, Centrale Marseille, Institut Fresnel, UMR 7249, 13397, Marseille, France to the fields acquisitions, the calibration stage and the noise measurements. All of these aspects are explained in Section II. Moreover, a non-linear inversion scheme, briefly described in Section III, must be employed where each data point is balanced according to its own accuracy in the inversion procedure. The resulting reconstructions are then provided in Section IV. Concluding remarks follow in Section V.

\section{Measurement CONFiguration}

\section{A. Target}

The target is the extremity of an absorbing material which is traditionally used to cover the walls of anechoic chambers (Figure 1(b)). This absorber is a foam composed of a polyurethane matrix mainly charged with carbon particles [13], thus it presents a complex relative permittivity $\varepsilon_{r}=\varepsilon_{r}^{\prime}+j \varepsilon_{r}^{\prime \prime}$. In particular, its imaginary part is finite and non-zero. In terms of size, its larger dimension is $70 \mathrm{~mm}(\sim 4 \lambda$ at $18 \mathrm{GHz})$. It is a very challenging target to measure. Indeed, the mean of the measured magnitude levels - on all the source/receiver positions - is about $60 \mathrm{~dB}$ lower than the incident wave inside the target.

\section{B. Experimental setup and measurement protocol}

The measurements were performed in the faradized anechoic chamber $(14.5 \times 6.5 \times 6.5) \mathrm{m}^{3}$ of the CCRM in Marseille (Figure 1(a)), already described in various papers [5], [7]. The different mechanical movements - transmitting antenna, target support, receiving antenna - allow the field measurements in a multistatic configuration almost all around a sphere surrounding the target. The microwave equipment is based on a network analyzer (HP8510) with two external mixers and two external synthesizers. The transmitting and the receiving antennas are standard horn antennas (ARA MWH 126B) and all the measurements are performed at $18 \mathrm{GHz}$. The emitter has a polarization along the $\mathbf{e}_{\phi}$ vector and the receiver sensitive to the polarization $\mathbf{e}_{\phi}$ - using the Bohren and Huffman convention.

The experimental scattered field is obtained from the complex difference between the total field measurement - acquired in presence of the target - and the incident field measurement - acquired without the target. Performing quantitative reconstructions requires an accurate calibration procedure. The scattered field measurements were then referred to an incident field with a magnitude of 1 and a null phase at the origin of the coordinate system. For this, a reference field, in the presence of a perfectly known target - here a metallic sphere was also measured. A complex-valued calibration coefficient 


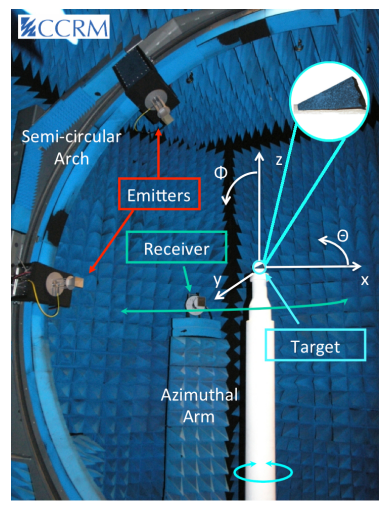

(a) Spherical setup

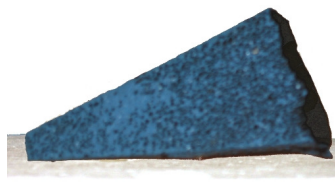

(b) Piece of a microwave absorber
Fig. 1. Pictures of the anechoic chamber setup and of the target

was computed by comparison with Mie series [5], [7] (one coefficient for each frequency).

As many measurements have to be acquired, drift phenomena can have a particular dramatic effect. Indeed, the scattered field - obtained from the difference between two fields - is greatly sensitive to any kind of disturbances. To compensate for the drift errors, we take advantage of the angular spectral properties of the scattered field [15]. The scattering measurements were therefore post-treated with a correcting procedure based on the fact that the propagation has a low-pass filter behavior (details on this procedure can be found in [16]).

\section{INVERSION PROCEDURE}

The purpose of the imaging procedure is to recover the complex permittivity map of an investigation zone from our scattered field measurements. In this paper, the investigation zone is limited to the center of the spherical setup, but its position and size can directly be deduced from the measurements [8]. An iterative scheme is adopted to solve this inverse scattering problem. At each step, the real part and the imaginary part of the permittivity are optimized by minimizing a cost-function.

We have chosen to base our permittivity reconstruction algorithm on a Bayesian formulation [20], where the density of probability of the unknown relative permittivity map $\varepsilon_{r}$ while knowing the scattered field measurement $\mathbf{E}_{\mathbf{s}}^{\mathbf{m}}$ reads

$$
p\left(\varepsilon_{r} \mid \mathbf{E}_{\mathbf{s}}^{\mathbf{m}}\right)=\frac{p\left(\varepsilon_{r}\right) p\left(\mathbf{E}_{\mathbf{s}}^{\mathbf{m}} \mid \varepsilon_{r}\right)}{p\left(\mathbf{E}_{\mathbf{s}}^{\mathbf{m}}\right)}
$$

When no statistical information is further introduced, maximizing the density of probability $p\left(\varepsilon_{\mathbf{r}} \mid \mathbf{E}_{\mathbf{s}}^{\mathbf{m}}\right)$ leads to the Maximum Likelihood solution. As each data point is balanced according to its own accuracy, and as we assume no noise correlation from one measurement point to another due to the experimental protocol, the involved cost-function is [18]

$$
\begin{aligned}
F\left(\varepsilon_{r}\right)=\sum_{s=1}^{N_{s}} \sum_{r=1}^{N_{r}} \frac{\left(\operatorname{Re}\left[\mathbf{E}_{\mathbf{s}}^{\mathbf{m}}\left(\mathbf{r}_{s}, \mathbf{r}_{r}\right)-\mathbf{E}_{\mathbf{s}}^{\mathbf{c}, \varepsilon_{\mathbf{r}}}\left(\mathbf{r}_{s}, \mathbf{r}_{r}\right)\right]\right)^{2}}{\sigma_{R e}^{2}\left(\mathbf{r}_{s}, \mathbf{r}_{r}\right)} \\
+\frac{\left(\operatorname{Im}\left[\mathbf{E}_{\mathbf{s}}^{\mathbf{m}}\left(\mathbf{r}_{s}, \mathbf{r}_{r}\right)-\mathbf{E}_{\mathbf{s}}^{\mathbf{c}, \varepsilon_{\mathbf{r}}}\left(\mathbf{r}_{s}, \mathbf{r}_{r}\right)\right]\right)^{2}}{\sigma_{I m}^{2}\left(\mathbf{r}_{s}, \mathbf{r}_{r}\right)}
\end{aligned}
$$

with $\sigma_{R e}$ (respectively $\sigma_{I m}$ ) the noise standard deviations on the real (respectively imaginary) part of the scattered field and $N_{s}$ (respectively $N_{r}$ ) the numbers of sources (respectively receivers). The noise standard deviations correspond to the real random noise disturbing the measurements. This noise, previously characterized, is strongly different from one measurement point to another [7]. Indeed, the real and imaginary parts of the random noise can be assumed to follow two Gaussian laws with standard deviations depending on the magnitude of the incident field, on the magnitude of the total field and on the frequency. Previous studies have shown that accurately determining and introducing $\sigma_{R e}$ and $\sigma_{I m}$ in (Eq. 3) is a pre-requisite for successfully reconstructing quantitative permittivity maps [18]. This aspect is of particular importance when dealing with this type of weakly scattering target.

The minimization was performed with a Polak-Ribiere conjugate gradient algorithm [22]. Classical cartesian basis functions were used to described the permittivity map and a positivity constraint was added to this permittivity map by taking as unknowns $\xi$ and $\eta$, so that $\varepsilon_{r}^{\prime}=1+\xi^{2}$ and $\varepsilon_{r}^{\prime \prime}=\eta^{2}$.

As the considered target is weakly contrasted, the inverse problem was linearized using the so-called Born approximation. This allows to reduce the memory requirement and to speed-up the inversion. A comparison between these reconstructed maps and the ones obtained without Born approximation shows that this linearization leads to less than $0.2 \%$ of variations on the reconstructed maps.

\section{IMAGING RESULTS}

Unfortunately, it is not possible to measure all the available information on the target [14] in a reasonable amount of time. We chose to keep the duration of the measurement inferior to 24 hours and this leads to 14 sources positions, 9 target orientations and 53 receivers positions with our setup. All angular steps were chosen with respect to a minimal spatial sampling angle which ensures independent measurement points. This corresponds to only $20 \%$ of the total number of information on the target in far-field.

The following reconstruction was obtained from the measured scattered field while considering an investigation domain of $(100 \times 100 \times 100) \mathrm{mm}^{3}$ centered at the origin. The number of measured points corresponds thus to $1.3 \%$ of the number of unknowns.

The shape of the absorbing material was clearly reconstructed as it can be seen on the 3D iso-surface images presented in [21] where the Born approximation was not applied in the inversion process. To deepen our study and to reach real quantitative information of the inner composition of our sample, we have made a finer analysis which is presented here with plane cross-sections passing through the "middle" of the target plotted in Figure 2. The red line corresponds to the laser beam used during the positioning procedure of the target. The absorber is well reconstructed in the entire search volume. Small variations appear in the permittivity map, corresponding most probably to structural inhomogeneities. It can be noticed that the reconstructed values of the permittivity are very weak, in particular the values of the real part. For a countermeasure, a 

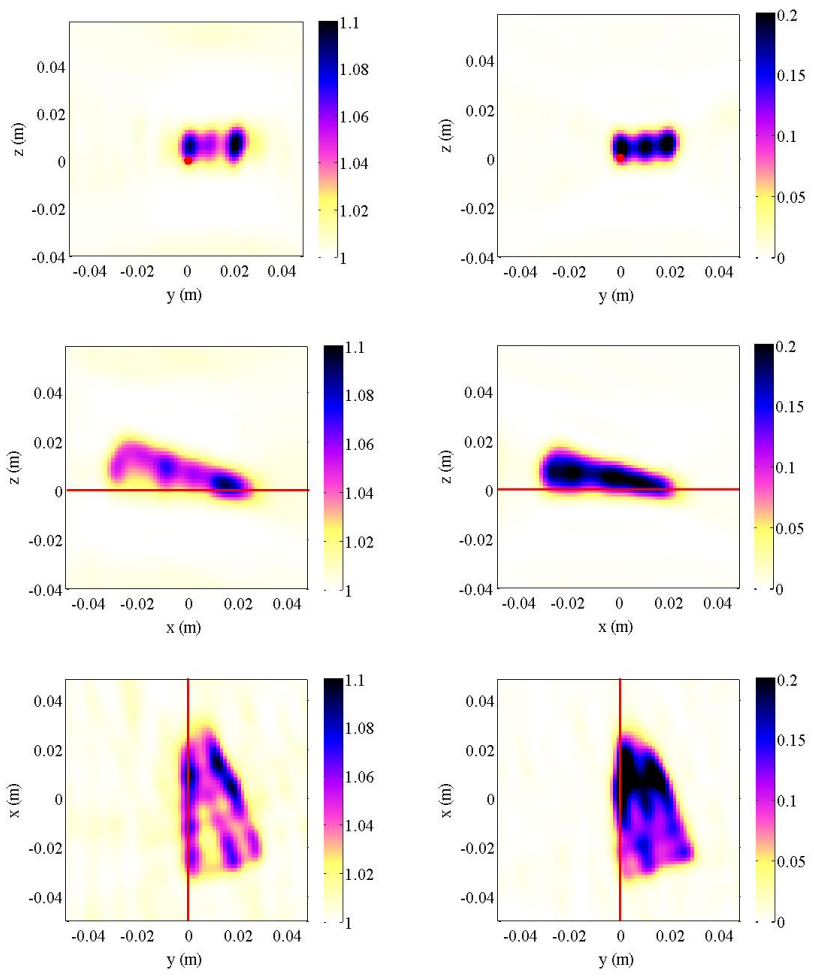

Fig. 2. Reconstructed permittivity map - left: real part and right: imaginary part - at $18 \mathrm{GHz}$ in plane cross-sections. Top: in the $x=0 \mathrm{~mm}$ plane, middle: in the $y=11 \mathrm{~mm}$ plane, bottom: in the $z=-7.9 \mathrm{~mm}$ plane.

spherical sample was machined from the same sort of absorber. The permittivity value of this spherical sample - assumed to be homogeneous - was determined by directly comparing the measurements with computations based on Mie series [23]. Its permittivity value was found to be equal to $\varepsilon_{r}=1.2+j 0.2$ at $18 \mathrm{GHz}$. This permittivity value is thus completely consistent with the permittivity values reconstructed herein.

\section{CONClusion}

In this paper, we have shown our capacity to quantitatively characterize the inner composition of a very weak scatterer from its measured scattered field. This object is an heterogeneous lossy target and the deduced permittivity values have been confirmed thanks to countermeasures. Imaging a fragment of absorber was possible because the following conditions were met: highly accurate measurements, an adequate estimation of the residual noise, a balance of each measurement point with respect to its own accuracy during the inversion algorithm and an efficient non-linear inverse scattering scheme.

Future works will concern the uncertainty quantification of the reconstruction maps. An analysis of the structure heterogeneities will also be carried out. The level of maturity of this microwave imaging instrument is such that we can now examine natural targets with complex features.

\section{ACKNOWLEDGMENT}

The authors wish to acknowledge B. Lacroix from the CETHIL laboratory, Lyon-France, for all the mechanics involving the fabrication of the absorber sphere.

\section{REFERENCES}

[1] A. Litman and L. Crocco, Guest Editor introduction, Inverse Problems 26:020201, 2009.

[2] M. Brignone, G. Bozza, A. Randazzo, M. Piana, M. Pastorino, A Hybrid Approach to 3D Microwave Imaging by Using Linear Sampling and ACO, IEEE Trans. Ant. Prop. 56:3224-3232, 2008.

[3] M. Donelli, D. Franceschini, P. Rocca and A. Massa, Three-Dimensional microwave imaging problems solved through an efficient multiscaling Particle Swarm Optimization, IEEE Trans. Geosc. Rem. Sens. 47:14671481, 2009

[4] K. Yang, J. Zhou and M. Tong, Inversion of electromagnetic scattering for $3 D$ dielectric objects through integral equation method with Nystrm discretization, IEEE Trans. Ant. Prop. 61:3387-3392, 2013.

[5] C. Eyraud, J.-M. Geffrin, P. Sabouroux, P. Chaumet, H. Tortel, H. Giovannini and A. Litman, Validation of a $3 D$ bistatic microwave scattering measurement setup, Radio Science 43:RS4018, 2008.

[6] J.-M. Geffrin and P. Sabouroux, Continuing with the Fresnel database: Experimental setup and improvements in $3 D$ scattering measurements, Inverse Problems 25:024001, 2009.

[7] J.-M. Geffrin, C. Eyraud, A. Litman and P. Sabouroux, Optimization of a bistatic microwave scattering measurement setup: From high to low scattering targets, Radio Science 44:RS003837, 2009.

[8] C. Eyraud, J.-M. Geffrin, A. Litman and J.-P. Spinelli, A large 3D target with small inner details: A difficult cocktail for imaging purposes without a-priori knowledge on the scatterers geometry, Radio Science 47:RS0E23, 2012.

[9] J. de Zaeytijd, A. Franchois and J.-M. Geffrin, A new value picking regularization strategy-application to the 3-D electromagnetic inverse scattering problem, IEEE Trans. Ant. Prop. 57:1133-1149, 2009.

[10] M. Haynes, J. Stang and M. Moghaddam, Real-time microwave imaging of differential temperature for thermal therapy monitoring, IEEE Trans. Biomed. Eng. PP:99, 2014.

[11] Chun Yu, Mengqing Yuan, Yangjun Zhang, J. Stang, R.T. George, G.A. Ybarra, W.T. Joines, and Q.H. Liu, Microwave imaging in layered media: 3-D image reconstruction from experimental data, IEEE Trans. Ant. Prop. 58:440-448, 2010.

[12] T. Grzegorczyk, P. Meaney, P. Kaufman, R. di Florio-Alexander and K. Paulsen, Fast 3-D tomographic microwave imaging for breast cancer detection, IEEE Trans. Medical Imaging 31:1584-1592, 2012.

[13] G. Dun, Modélisation et optimisation de chambres anéchoiques pour applications CEM, PhD thesis, Ecole Nationale Supérieure des Télécommunications de Bretagne, 2007.

[14] O. Bucci and T. Isernia, Electromagnetic inverse scattering: retrievable information and measurement strategies, Radio Science 32:2123-2137, 1997.

[15] O. Bucci and G. Franceschetti, On the spatial bandwidth of scattered fields, IEEE Trans. Ant. Prop. 35:1445-1455, 1987.

[16] C. Eyraud, J.-M. Geffrin, P. Sabouroux and H. Giovannini, Drift correction for scattering measurements, Appl. Phy. Lett., 89:244104, 2006.

[17] B. Barrowes, L. Teixeira and J. Kong, Fast algorithm for matrixvector multiply of asymmetric multilevel block-toeplitz matrices in 3-D scattering, Microwave Optical Tech. Lett. 31:28-32, 2001.

[18] C. Eyraud, A. Litman, A. Hérique and W. Kofman, Microwave imaging from experimental data within a Bayesian framework with realistic random noise, Inverse Problems, 26, 024005, 2009.

[19] O. Merchiers, C. Eyraud, J.-M. Geffrin, R. Vaillon, B. Stout, P. Sabouroux and B. Lacroix, Microwave measurements of the full amplitude scattering matrix of a complex aggregate: a database for the assessment of light scattering codes, Optics Express 18:121191, 2010.

[20] G. Demoment and J. Idier, Problèmes inverses : de l'expérimentation à la modélisation: Approche bayésienne pour la résolution des problèmes inverses en imagerie, $\mathrm{M}$. Bonnet ed., Observatoire français des techniques avancées, Paris, 59-77, 2001.

[21] J.-M. Geffrin, C. Eyraud, A. Litman, 3D Inversion of Lossy Targets From Free Space Scattering Measurements, Proc. of European Conference on Antennas and Propagation (Eucap), 3699 - 3702, 2012.

[22] E. Polak, Computational methods in optimization, Acad. Press, 1971.

[23] C. Eyraud, J.-M. Geffrin, A. Litman and H. Tortel, Complex permittivity determination from far-field scattering patterns, IEEE Antennas and Wireless Propagation Letters 14(1): 309-312, 2015. 